\title{
Desempeño digital de los docentes desde la emergencia sanitaria del COVID-19
}

Teachers digital performance since the COVID-19 health emergency

\author{
Ruth Eleana Herrera-García \\ rutheleana.herrerag@gmail.com \\ Universidad Cesar Vallejo, Chimbote \\ Perú \\ https://orcid.org/0000-0002-6936-1547 \\ Ana María Vargas-Castillo \\ vargascas5018@gmail.com \\ Universidad Cesar Vallejo, Chimbote \\ Perú \\ https://orcid.org/0000-0003-2045-8265 \\ Mery Marleny Martínez-lbarra \\ merymarlenymartinezibarra1@gmail.com \\ Universidad Cesar Vallejo, Chimbote \\ Perú \\ https://orcid.org/0000-0001-9714-063X \\ Ángela Beatriz Rodríguez-Montes \\ angelarodriguezmontes3@gmail.com \\ Universidad Cesar Vallejo, Chimbote \\ Perú \\ https://orcid.org/0000-0001-8404-1763
}

Recepción: 10 de junio 2021

Revisado: 15 de julio 2021

Aprobación: 01 de septiembre 2021

Publicación: 15 de setiembre 2021 


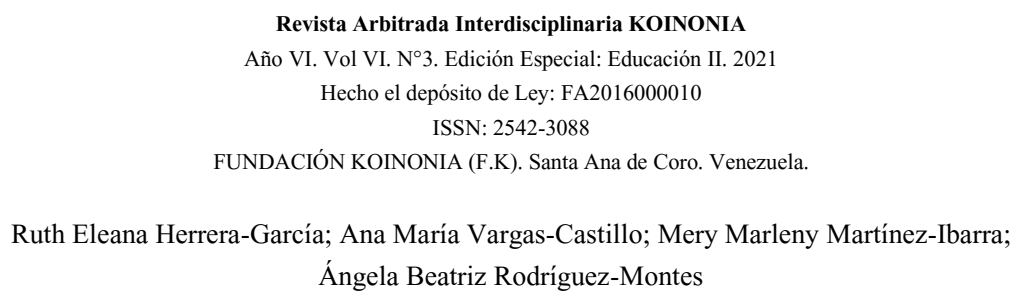

\title{
RESUMEN
}

Este es un momento para significar el desempeño docente en tiempos de pandemia por Covid19, contexto para el cual no se estaba preparado. De allí que, se buscó analizar el desempeño que han tenido los docentes del Distrito Nuevo Chimbote, en el marco del programa Aprendo en Casa implementado en el Perú. Metodológicamente, el estudio se desplegó desde un enfoque cuantitativo, caracterizándose por ser de campo, descriptivo y de corte transversal. La muestra poblacional estuvo constituida por 1246 estudiantes, seleccionados mediante muestreo por cuota. Los datos se recabaron mediante un cuestionario de preguntas cerradas y se procesaron estadísticamente. Para abordar contextos educativos y desarrollar escenarios de aprendizaje en situaciones adversas como las actuales, necesariamente, el docente debe desplegar acciones concretas que lo muestren como un profesional de vanguardia, involucrado con las tecnologías, debe saber gestionar espacios de interrelación, buena escucha y diálogo que coadyuven al desarrollo socioemocional de los estudiantes.

Descriptores: Docente, desempeño, tecnologías. (Palabras tomadas del Tesauro UNESCO).

\begin{abstract}
This is a moment to signify the teaching performance in times of the Covid19 pandemic, a context for which it was not prepared. Hence, it was sought to analyze the performance of the teachers of the Nuevo Chimbote District, within the framework of the Learning at Home program implemented in Peru. Methodologically, the study was developed from a quantitative approach, characterized by being field, descriptive and cross-sectional. The population sample consisted of 1246 students, selected by quota sampling. Data were collected using a closed-question questionnaire and statistically processed. To address educational contexts and develop learning scenarios in adverse situations such as the current ones, necessarily, the teacher must deploy concrete actions that show him as a cutting-edge professional, involved with ICT, he must know how to manage spaces of good listening and dialogue that contribute to the socio-emotional development of students.
\end{abstract}

Descriptors: Teacher, performance, technologies. (Words taken from the UNESCO Thesaurus). 


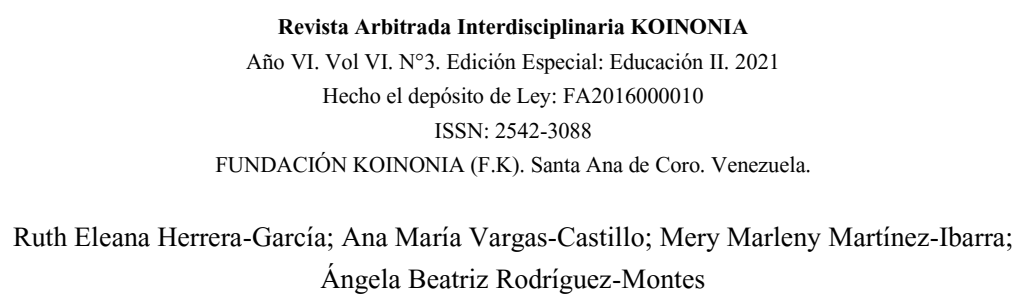

\section{INTRODUCCIÓN}

Actualmente la educación peruana afronta muchos retos sobre todo lo que respecta a la actuación que debe evidenciar el docente; pues, cada vez se exige que el magisterio responda a los cambios que continuamente se vienen presentando en la educación. La sociedad demanda de los maestros acciones que permitan garantizar el logro de los aprendizajes, sin embargo, esta exigencia no llega a ser coherente; ya que, por un lado, el Estado pareciera no ofrece las condiciones suficientes que permitan elevar la profesionalidad docente, ya sea brindando facilidades para estudios de postgrados, intercambio de experiencias, entre otros. Por otro lado, la creencia de los padres de familia, que han convertido las instituciones en una especie de guardería donde el docente tiene que hacerse cargo de los estudiantes sin recibir mucho apoyo por parte de estos, bajo el pretexto del trabajo.

De allí que uno de los temas más cruciales de abordaje en el campo pedagógico y educativo está relacionado con el desempeño docente, considerando que este, como acción humana determinante del proceso pedagógico, son actitudes y modos que están interrelacionadas tanto a la preparación inicial de la carrera docente, como a la experiencia cotidiana, a fin de alcanzar los niveles esperados en educación.

Ahora bien, para comprender mejor el tema es menester cavilar en torno a que el desempeño docente está entretejido con la forma de actuación práctica, pues, como expresan Martínez, Guevara \& Valles (2016), desde lo enunciado por Ponce (2005), son actuaciones concretas llevadas a cabo por estos, durante el proceso educativo y desde sus competencias pedagógicas, para obtener un resultado positivo en el aprendizaje. Al mismo tiempo, estás acciones están inextricablemente unidas a la disposición del profesor como ente que media en los educandos, pues concierne su aptitud y poder de decisiones en contextos educativos, que conllevan a la realización de actividades y tareas guiadoras de la formación de estudiantes competentes para participar y transformar su entorno socio-cultural (Flores, 2008).

En este contexto, se puede traer a colación a Consuegra (2010) \& MINEDU (2012), cuando expresan que el desempeño docente se evidencia en las actuaciones concretas que ponen de manifiesto el talento y competencia del profesor en el 


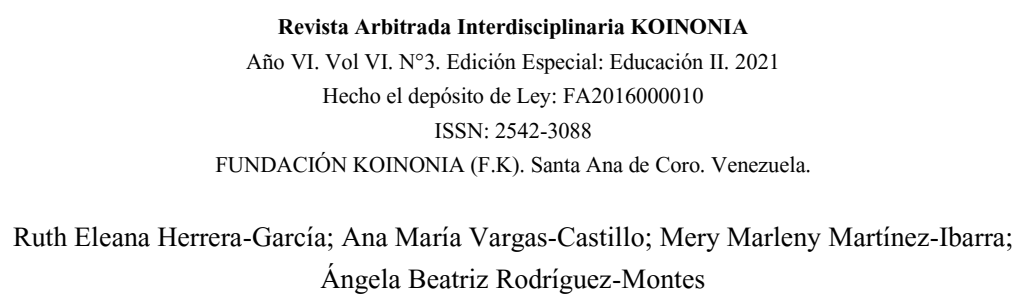

desarrollo de su práctica educativa cotidiana; pues, desde su interior emerge un potencial innato, una motivación para actuar con capacidad y habilidad, que debe ser explotada para alcanzar las necesidades más elevadas. En otras palabras, todas las potencialidades que se poseen se inician en el interior del ser y se consolidan con conductas y comportamientos idóneos para el logro de la satisfacción interna y la autorrealización personal.

Lo planteado hasta ahora, hace entender que el desempeño docente es una acción que, aunque emerja entrelazada a múltiples factores como los fines educativos, el estudiante y el entorno en general, está determinada esencialmente por elementos coligados a la propia interioridad del docente. Tal es el caso de que entre la mediación motivación-desempeño docente, es determinante la percepción que tiene él de su propia capacidad de lograr una meta con éxito, así, su autoeficacia se ve continuamente enfrentado a nuevos retos. Por lo contrario, los docentes que se sienten ineficaces se ven debilitados, estresados y desarrollan actividades menos efectivas con sus estudiantes; pues, el comportamiento es regulado por las expectativitas de eficacia y la convicción que se tiene de tener éxito, para alcanzar los objetivos esperados (Covarrubias \& Mendoza, 2013)

En este escenario de nuevos retos que representa el giro pedagógico que ha tenido que dar la educación dada la situación de pandemia por Covid19 declarada desde marzo de 2020 y que actualmente sigue viviendo el planeta, el tema de desempeño docente ha sido decisivo. Aquí, cabe recordar que Perú fue el primer país de América Latina que decreto el distanciamiento social obligatorio, generando con ello un impacto en la educación de 9,9 millones de estudiantes peruanos al anunciarse una nueva estrategia de educación a distancia. Así, la emergencia sanitaria fue determinante para darle un vuelco a como venía desarrollando el profesor su práctica educativa en la cotidianidad; pues fue, y sigue siendo necesario, establecer nuevas formas didácticas de enseñanza-aprendizaje. Situación que ha incidido de una u otra manera en su accionar y, con ello, en las interrelaciones que se dan, tanto con estudiantes, como con padres y familiares. 


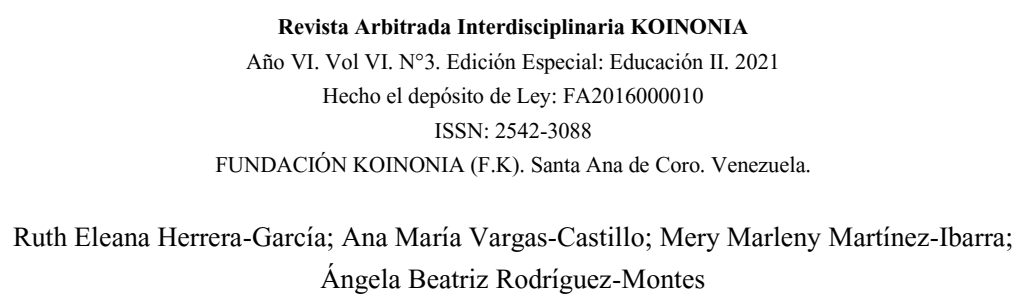

Desde este contexto, resulta bien significativo concatenar el desempeño docente a tres factores o dimensiones que han sido vitales para el desarrollo del aprendizaje en el marco del programa Aprendo en Casa implementado por el Sistema Educativo peruano a raíz de la pandemia. Dimensiones que desde la percepción del estudiante son importantes, pues, engloban una actitud de compromiso personal; y, como exponen Gálvez \& Milla (2018), solo con compromiso los docentes demostrarán mejores desempeños, por tanto, los aprendizajes escolares también serán buenos.

En primer término, la educación online ha tomado un rol protagónico, ya que, el proceso de aprendizaje se ha centrado en el uso de recursos tecnológicos y entornos virtuales de aprendizaje; lo cual, desde lo expresado por Valdivieso \& Gonzales (2016) demanda que el docente cuente con las condiciones necesarias para desplegar aptitudes y capacidades en el uso de la tecnológica y herramientas virtuales en su práctica diaria.

En vista de esto, el gobierno, al instalar la plataforma web y el programa Aprendo en Casa, buscó realizar las adaptaciones necesaria, con el fin de que los docentes mejorasen su desempeño en el quehacer educativo; sin embargo, en un inicio, este no se encontraba preparado, tenía mucho desconocimiento tecnológico, pero, con el tiempo muchos asumieron el compromiso de lograr los retos. Han innovado y desplegado su práctica haciendo uso de variadas estrategias y recursos basados en la tecnología, que, como expresan Cabañas \& Ojeda (2003), pretenden que la experiencia de aprendizaje a distancia sea productiva y "brinde la posibilidad de que cada individuo moldee su propia forma de aprendizaje a partir del acceso a contenidos globales, desarrollando su capacidad crítica, comunicativa y reflexiva, sin ataduras físicas o temporales" (p. s/n).

Lo planteado, conlleva a visibilizar las acciones en pro del desarrollo socioemocional como segunda dimensión asociada al desempeño docente, dado el soporte que se ha tenido que dar a los estudiantes afectados por motivos diversos debido a la pandemia. Tal es el caso, que en esta situación de confinamiento, entendida como una medida urgente que toma el gobierno limitando el desplazamiento de las personas y cerrando o restringiendo el uso de diversos establecimientos, ya sean de diversión, culturales, 


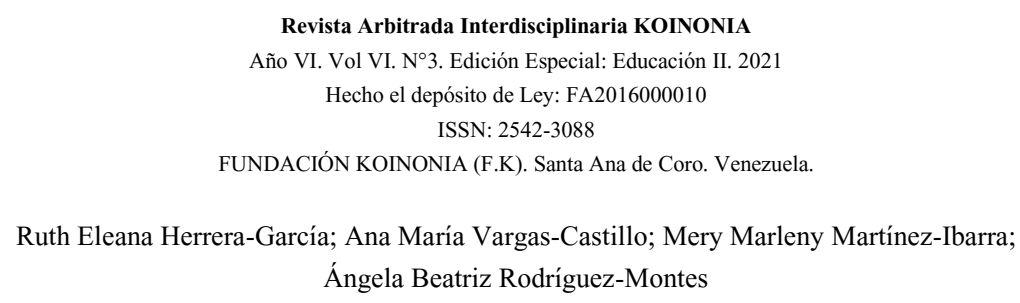

turístico, educativos, el docente ha tenido que gestionar y fortalecer espacios comunicacionales y de diálogo con estudiantes a fin de orientar y guiar las diversas situaciones sociales, psicológicas y emocionales que perturban el proceso de educación a distancia. Esto, en palabras de Goleman (1996), significa que el docente debe, con inteligencia emocional, saber gestionar las relaciones personales; es decir, deben tener habilidades sociales que los llevan a tener excelentes relaciones con los otros, las cuales son indispensables no solo para un buen desempeño laboral, sino también para coadyuvar a la felicidad personal en el entorno familiar y social, tanto de los otros como de sí mismo.

Aquí, cabe resaltar que la formación de grupos de entornos virtuales surge para dar respuesta a las necesidades no solo educativas, sino, también, socioemocionales de los estudiantes. Pues, estas últimas no tienen fronteras y se escenifica en múltiples formas: ansiedad, duelos, hipocondrías, depresiones, trastornos evitativos, fobias, trastornos del sueño. Por tanto, es importante que en el aula virtual tenga mecanismos de comunicación fluida entre docentes, alumnos, padres de familia, para que se dé como enuncia Rizo (2020), un compromiso e involucramiento positivo de los estudiantes en las actividades de acompañamiento pedagógico y de producción de saberes, en estos escenarios de aprendizajes .

Por último, el trato a los padres de familia desde una actitud de escucha se presenta como una competencia actitudinal y acción elemental del desempeño de los docentes interrelacionada al pro del desarrollo socioemocional y del aprendizaje de los estudiantes. En este tenor, Rice (2011) \& Laverty (2011), referenciado por Motta-Ávila (2017) expresan que la concepción aristotélica de la buena escucha, se constituye en una virtud que juega un papel preponderante en la comunicación humana, pues, es en este acto, que los seres humanos satisfacen o fracasan en el imperativo de entender a los demás.

De allí que, el docente debe establecer y fomentar una relación positiva con los padres de familia, a propósito de que estos puedan favorecer una actitud pro social en el contexto familiar, pues, esto influye marcadamente en la conducta y buen desarrollo de los hijos (Garaigordobil, 2014). Promover conductas pro sociales que conlleven a 


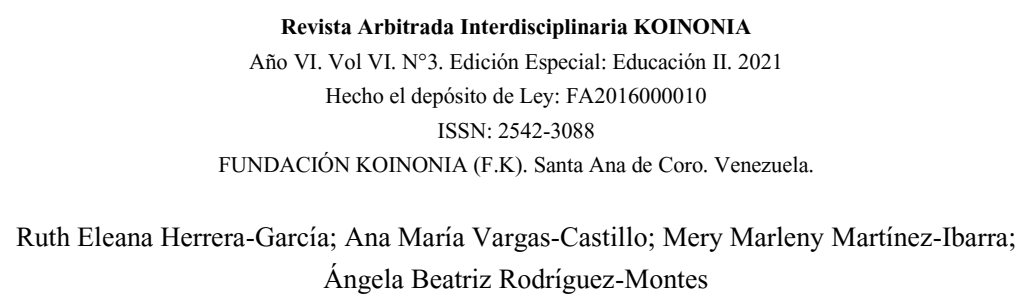

la adquisición de confianza para relacionarse con los demás y favorezcan la formación de los estudiantes, se da en la medida que estos sean orientados positivamente por los padres (Aguilar, 2014).

Lo expuesto, en palabras de Martínez, Esparza \& Gómez (2020), significan que el desempeño docente esta consustanciado fundamentalmente; primero, a la percepción que tiene el docente de su ser sí mismo como profesional, para enfrentar los retos y desafíos que se le presentan en su práctica educativa; segundo, a la capacidad de innovar pedagógicamente para transformar las menguas en ventajas, jugando aquí un papel importante el involucramiento con las tecnologías de la información y la comunicación (TIC), como forma de diversificar los recursos didácticos y motivar al aprendizaje; $y$, por último, a su poder de liderazgo para involucrarse en las relaciones personales con los distintos sujetos participantes del proceso y, así, influir en el desarrollo de conductas y comportamientos socioemocionales favorables en el estudiante.

En virtud de lo planteado, se buscó desarrollar una investigación sobre el desempeño, que han tenido los docentes que ejercen en el Distrito Nuevo Chimbote, departamento de Áncash del Perú. A razón de ello, fue menester cuestionar desde la perspectiva del estudiante de Educación Básica ¿Cómo ha sido el desempeño de los docentes que ejercen en el Distrito Nuevo Chimbote, en los actuales momentos de pandemia por Covid19? Pues, esto permitió desarrollar la investigación encausada en analizar el desempeño que han tenido los docentes del Distrito Nuevo Chimbote, en el escenario de pandemia por Covid19.

\section{METODOLOGÍA}

Es de saber que en este esté apartado se disponen las definiciones metodologías guiadoras del proceso investigativo desarrollado en relación al desempeño que han tenido los docentes del Distrito Nuevo Chimbote en el marco del programa Aprendo en Casa implementado por el Sistema Educativo peruano a raíz de la pandemia.

En tal sentido, el estudio se desplegó desde un enfoque cuantitativo y, para responder al problema de investigación sin manejo deliberado de ninguna variable, se asumió un 


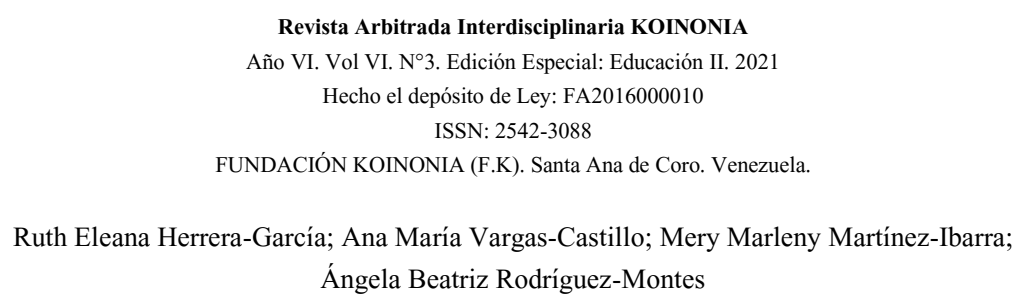

diseño no experimental. Cabe resaltar, que este tipo de diseño hizo que la misma se constituyera en un estudio de campo, desarrollado a nivel descriptivo y de corte transversal. Ya que, la recolección de los datos primarios sobre los hechos o fenómenos se realizó directamente de la realidad donde ocurren, en un período único y con el propósito de describirlos tal cual como acontecen (Palella \& Martins, 2015). La muestra poblacional, estuvo constituida por 490 estudiantes de Inicial, 573 de Primaria y 183 de Secundaria, para un total de 1246 estudiantes, seleccionados mediante la técnica muestral por cuota, dado que se prefijo como criterio que fuesen estudiantes del Distrito de Nuevo Chimbote, pertenecientes a los niveles de Inicial, Primaria y Secundaria que estructuran la Educación Básica peruana. No obstante, la elección de las unidades de cada nivel se realizó se realiza sin atender a ningún procedimiento de selección (Palella \& Martins, 2015).

En cuanto a las técnicas e instrumentos para recabar los datos se utilizó una encuesta operacionalizada mediante un cuestionario formulado en línea, de carácter anónimo para los estudiantes de los 3 niveles. Cabe decir, que dicho instrumento se estructuro con 20 preguntas para los estudiantes de primaria y secundaria y 10 preguntas para los estudiantes de inicial, de tipo cerradas y con respuestas estructuradas mediante una escala de valores, las cuales debían ser respondidas en conjunto con los padres de familia.

El procesamiento de los datos se realizó mediante la estadística descriptiva y se presentan a través de tablas con sus respectivos análisis (Palella \& Martins, 2015). Es de saber que la validez del instrumento se dio mediante la técnica de juicios de expertos; en tal sentido, la revisión de contenido, redacción y pertinencia hecha a cada ítem permitió hacer las correcciones pertinentes. Asimismo, la confiabilidad del instrumento se estableció a través del Coeficiente de Alfa de Cronbach, el cual es un indicador de la consistencia interna de los ítems. Para ello, se aplicaron 3 pruebas piloto, una por cada nivel, arrojando un $r=0.923$, lo que indica que es un instrumento de muy alta confiabilidad.

\section{RESULTADOS}




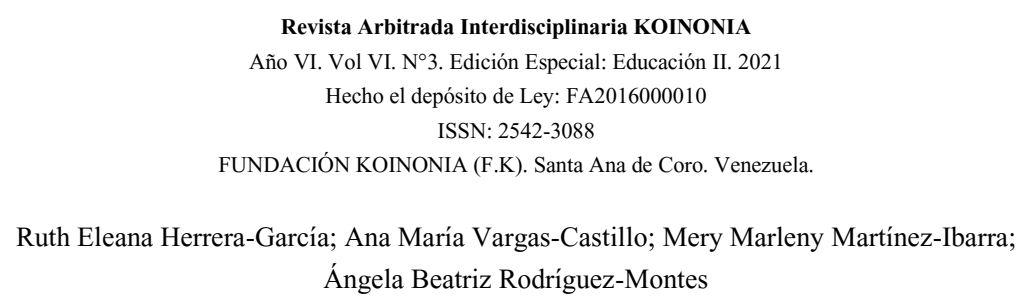

En este punto se presentan los resultados obtenidos en la encuesta aplicada a 1246 estudiantes del Distrito de Nuevo Chimbote, a fin de describir el desempeño que han tenido los docentes, en el contexto del programa Aprendo en Casa desarrollado para atender la emergencia sanitaria por Covid19. De allí que:

Con respecto a cómo perciben el desempeño docente, los estudiantes de Educación Inicial, se tiene:

Tabla 1.

Percepción del desempeño docente por parte de los estudiantes de Educación Inicial en el escenario de pandemia por Covid19.

\begin{tabular}{|c|c|c|c|c|}
\hline DIMENSIONES & BAREMO & FRECUENCIA & $\begin{array}{l}\text { PORCENTAJE \% } \\
\text { POR NIVEL }\end{array}$ & $\begin{array}{l}\text { PORCENTAJE QUE REPRESENTA } \\
\text { EN LA TOTALIDAD DE LA } \\
\text { MUESTRA POBLACIONALDE } 1246 \\
\text { ESTUDIANTES }\end{array}$ \\
\hline TRATO A LOS & EXDD & 429 & 87.55 & 34,43 \\
\hline PADRES DE FAMILIA & MBDD & 27 & 5,51 & 2,16 \\
\hline MANEJO DE & BDD & 22 & 4,49 & 1,77 \\
\hline $\begin{array}{l}\text { RECURSOS } \\
\text { TECNOLÓGICOS }\end{array}$ & RDD & 7 & 1,43 & 0,56 \\
\hline $\begin{array}{l}\text { DESARROLLO } \\
\text { SOCIOEMOCIONAL }\end{array}$ & DDD & 5 & 1,02 & 0,41 \\
\hline TOTAL & & 490 & 100 & 39,32 \\
\hline
\end{tabular}

Elaboración: Los autores.

Los resultados de la tabla 1 muestran que el $97,55 \%$ de los estudiantes encuestados perciben que los maestros de Educación Inicial, con la ejecución del programa Aprendo en Casa, han tenido y tienen un desempeño que se puede describir de bueno a excelente, en contraposición al $2,45 \%$ de los estudiantes que manifiestan tener entre regular y deficiente desempeño docente. Cabe agregar que esta cuota de estudiantes representa el $39,32 \%$ de los estudiantes encuestados dentro de la totalidad de la muestra poblacional estimada. 
En relación a la percepción sobre el desempeño docente que tienen los estudiantes de Educación Primaria, se presenta:

\section{Tabla 2.}

Percepción del desempeño docente por parte de los estudiantes de Educación Primaria en el escenario de pandemia por Covid19.

\begin{tabular}{|c|c|c|c|c|}
\hline DIMENSIONES & BAREMO & FRECUENCIA & $\begin{array}{l}\text { PORCENTAJE \% } \\
\text { POR NIVEL }\end{array}$ & $\begin{array}{l}\text { PORCENTAAE QUE REPRESENTA } \\
\text { EN LA TTTALILADD DE LA } \\
\text { MUESTRA POBLACIONALDE } 1246 \\
\text { ESTUDIANTES }\end{array}$ \\
\hline TRATO A LOS & EXDD & 445 & 77,66 & 35,71 \\
\hline PADRES DE & MBDD & 99 & 17,28 & 7,94 \\
\hline FAMILIA & $\mathrm{BDD}$ & 15 & 2,62 & 1,21 \\
\hline $\begin{array}{l}\text { MANEJO DE } \\
\text { RECURSOS }\end{array}$ & RDD & 8 & 1,40 & 0,64 \\
\hline TECNOLÓGICOS & DDD & 6 & 1,04 & 0,48 \\
\hline \multicolumn{5}{|l|}{$\begin{array}{l}\text { DESARROLLO } \\
\text { SOCIOEMOCIONAL }\end{array}$} \\
\hline TOTAL & & 573 & 100 & 45,99 \\
\hline
\end{tabular}

Elaboración: Los autores.

Con los resultados de la tabla 2 se observa que el $97,56 \%$ de los estudiantes encuestados señalan que los maestros de Educación Primaria han tenido y tienen un desempeño docente tipificado de bueno a excelente en el marco del desarrollo del programa Aprendo en Casa, en contraste al 2,44\% de los estudiantes que declaran tener entre regular y deficiente desempeño docente. Es de saber, que esta cuota de estudiantes representa el $45,99 \%$ de los escolares encuestados en el conjunto que conforman la muestra poblacional atendida. 
En lo que respecta a la apreciación que tienen los estudiantes de Educación Secundaria sobre el desempeño docente, se observa:

Tabla 3.

Percepción del desempeño docente por parte de los estudiantes de Educación Secundaria en el escenario de pandemia por Covid19.

\begin{tabular}{|c|c|c|c|c|}
\hline DIMENSIONES & BAREMO & FRECUENCIA & $\begin{array}{l}\text { PORCENTAJE \% } \\
\text { POR NIVEL }\end{array}$ & $\begin{array}{l}\text { PORCENTAAE QUE REPRESENTA } \\
\text { EN LA TTTALILADD DE LA } \\
\text { MUESTRA POBLACIONALDE } 1246 \\
\text { ESTUDIANTES }\end{array}$ \\
\hline TRATO A LOS & EXDD & 102 & 55.74 & 8,19 \\
\hline PADRES DE & MBDD & 40 & 21,86 & 3,21 \\
\hline FAMILIA & BDD & 22 & 12,02 & 1,77 \\
\hline MANEJO DE & RDD & 10 & 5,46 & 0,80 \\
\hline TECNOLÓGICOS & DDD & 9 & 4,92 & 0,72 \\
\hline $\begin{array}{l}\text { DESARROLLO } \\
\text { SOCIOEMOCIONAL }\end{array}$ & & & & \\
\hline TOTAL & & 183 & 100 & 14,69 \\
\hline
\end{tabular}

Elaboración: Los autores.

Atendiendo a los resultados que se muestran en la tabla 3 y aunque se presenta una reducción un poco significativa de la percepción que tienen los estudiantes de Educación Secundaria en comparación con los de Educación Inicial y Primaria, se puede exponer que el $89,62 \%$ de los estudiantes encuestados señalan que los docentes, con la ejecución del programa Aprendo en Casa, han tenido y tienen un desempeño que se puede definir de bueno a excelente, a diferencia del 10,38\% de los estudiantes que indican tener un desempeño docente entre regular y deficiente. 


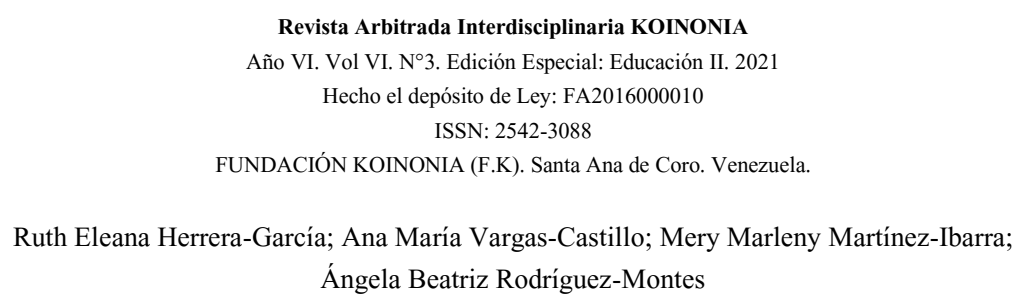

Se precisa que esta cuota de estudiantes representa el $14,69 \%$ de los estudiantes encuestados en la generalidad de la muestra poblacional estudiada.

Para cerrar, se presentan los resultados en torno a la percepción que tienen la totalidad de estudiantes encuestados sobre el desempeño que han tenido y tienen los docentes que ejercen en el Distrito de Nuevo Chimbote, en el marco del programa Aprendo en Casa desarrollado para atender la emergencia sanitaria por Covid19, el cual, se ha valorado desde las dimensiones: trato a los padres, manejo de recursos tecnológicos y desarrollo socioemocional como acciones concretas que muestran su desempeño. En tal sentido, se presenta la siguiente tabla:

\section{Tabla 4}

Desempeño del Docente que ejerce en Nuevo Chimbote en el escenario de pandemia por Covid19.

\begin{tabular}{|c|c|c|c|c|}
\hline VARIABLE & DIMENSIONES & BAREMO & FRECUENCIA & PORCENTAJE \% \\
\hline $\begin{array}{l}\text { DESEMPEÑO } \\
\text { DEL } \\
\text { DOCENTE } \\
\text { QUE EJERCE } \\
\text { EN NUEVO } \\
\text { CHIMBOTE }\end{array}$ & $\begin{array}{l}\text { Trato a los padres de } \\
\text { familia } \\
\text { Manejo de recursos } \\
\text { tecnológicos } \\
\text { Desarrollo socioemocional }\end{array}$ & $\begin{array}{l}\text { EXDD } \\
\text { MBDD } \\
\text { BDD } \\
\text { RDD } \\
\text { DDD }\end{array}$ & $\begin{array}{r}976 \\
166 \\
59 \\
25 \\
20\end{array}$ & \begin{tabular}{r|r|r|}
78,33 \\
13,32 \\
4,73 \\
2,01 \\
1,61
\end{tabular} \\
\hline & TOTAL & & 1246 & 100 \\
\hline
\end{tabular}

Elaboración: Los autores.

Con los resultados de la tabla 4 se observa que el 96,38\% (1.201 estudiantes) expresan que las acciones que han realizado y realizan los docentes de Nuevo Chimbote, hacen que su desempeño se estime de bueno a excelente, al mostrar sus talentos, desplegar sus capacidades y cumplir con sus competencias docentes, para desarrollar el proceso de aprendizaje en el marco del programa Aprendo en Casa implementado por el Sistema Educativo peruano a raíz de la pandemia. No obstante, también, se aprecia un porcentaje significativo de 3,6\% (45 estudiantes), que expresan 


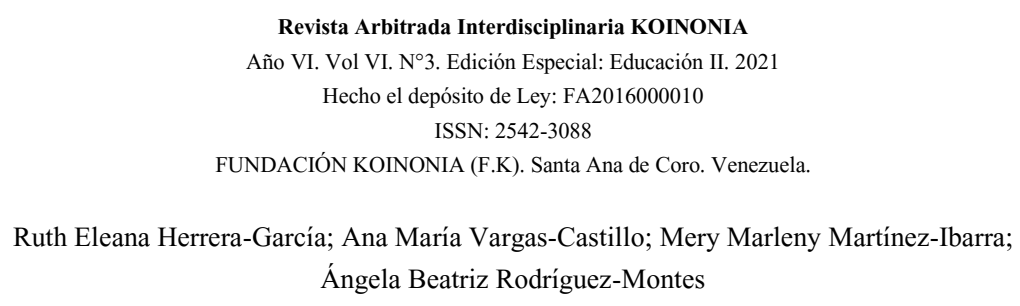

que el desempeño docente corresponde a regular y/o deficiente. Cabe resaltar, que estos resultados son indicativos de la globalidad de la muestra poblacional atendida.

\section{DISCUSIÓN}

Según los resultados obtenidos, se evidencia que los docentes de Nuevo Chimbote, que ejercen en los niveles de Educación inicial, primaria y secundaria, presentan entre un excelente a un buen desempeño desde la percepción de los actores involucrados, siendo el resultado de $96.38 \%$. Tales cifras, conllevan a considerar que los docentes han asumido, desde su compromiso docente, los contextos de cambios y los retos educativos demandados por el programa Aprendo en Casa, como forma de dar respuesta a las situaciones y circunstancias que se viven por la pandemia. Hallazgo que se compagina con lo expresado por: Consuegra (2010), MINEDU (2012), Covarrubias \& Mendoza (2013), Gálvez \& Milla (2018) y Martínez, Esparza \& Gómez (2020), en el sentido de que solo el docente comprometido desde su ser profesional, con expectativitas de eficacia y la convicción de tener éxito, podrá mostrar un desempeño constituido por sus mejores talentos y capacidades, para enfrentar los retos y desafíos que se le presentan en su práctica educativa cotidiana y lograr buenos aprendizajes en sus estudiantes.

Asimismo, los datos ponen en evidencia que, para un buen desempeño docente, es menester que este cuente con las condiciones y recursos que le permitan desplegar un conjunto de estrategias idóneas y certeras que conlleven a la producción de aprendizajes cónsonos para abordar las distintas realidades que se vive, sobre todo en estos momentos de emergencia sanitaria a causa de la pandemia. En tal sentido, la apropiación paulatina de los recursos tecnológico y la implementación de estrategias basadas en la virtualidad es y sigue siendo determinante para el proceso de enseñanza-aprendizaje de los estudiantes, aunque inicialmente representaba un reto, dada las carecías de conocimiento y practica que se exteriorizaron en este sentido. Idea que coincide con lo expresado por Valdivieso \& Gonzales (2016) y Cabañas \& Ojeda (2003), en torno a que es menester que el docente cuente con los conocimientos y herramientas tecnológicas que le permitan un desempeño idóneo en 


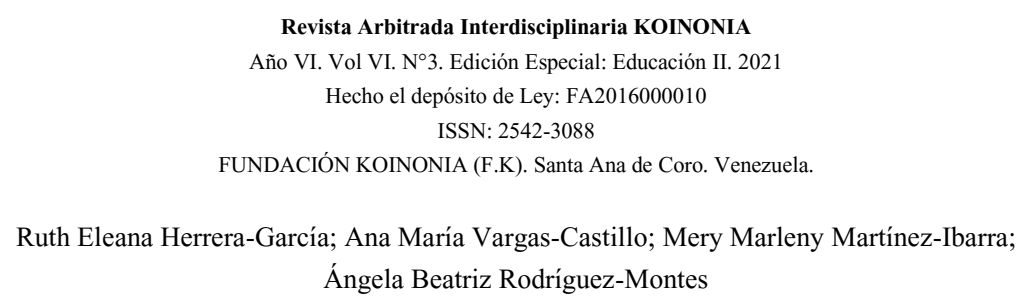

su práctica diaria, si se aspira que la experiencia de aprendizaje a distancia de los educandos se logre de manera productiva y enriquecedora.

Por otro lado, los hallazgos muestran que desplegar una estructura de acciones y comportamientos que soporten el desarrollo socioemocional es una dimensión imprescindible en el ámbito del desempeño docente, sobre todo en situaciones tan cruciales como las actuales de emergencia sanitaria. Las emociones estuvieron al tope, pues nadie estuvo preparado para enfrentar la pandemia, sin embargo, los docentes mostraron que son capaces de gestionar relaciones interpersonales, cualidad relacionada con la inteligencia emocional, tal como expresa Goleman (2006), y dar apoyo e involucrarse positivamente, no solo en las actividades de aprendizaje, sino, también, en momentos propiamente difíciles del acompañamiento pedagógico para realmente desarrollar saberes Rizo (2020).

Por último, los datos evidencian que un buen desempeño docente en situaciones adversas implica que estos muestren actitudes y modos para establecer alianzas con los padres de familia, para lo que es indispensable asumir la cualidad de saber escuchar y de diálogo, si se quiere tener éxito en el proceso de enseñanza aprendizaje tal como refieren Rice (2011) \& Laverty (2011), referenciados por Motta-Ávila (2017). Saber escuchar y dialogar, son factores elementales en una buena relación, entonces, los docentes deben coadyuvar a la creación de espacios comunicativos entre estudiantes y padres de familia, que conlleven al entendimiento y orienten sobre cómo debe ser sus reacciones frente a las diversas situaciones que se viven en la actual cotidianidad. Pues, una actitud de escucha y dialogo por parte de padres de familia y docentes influye marcadamente en la actitud y comportamiento de los estudiantes, coincidiendo esto con lo enunciado por Garaigordobil (2014).

\section{CONCLUSIONES}

El desempeño docente, aunque se funda desde la interioridad del ser docente y se moviliza desde su talento, aptitud y poder de decisiones, es un elemento que fundamentalmente se materializa con las actitudes y formas de actuación que despliega todo maestro $\mathrm{y} /$ profesor con su práctica cotidiana, para desarrollar 


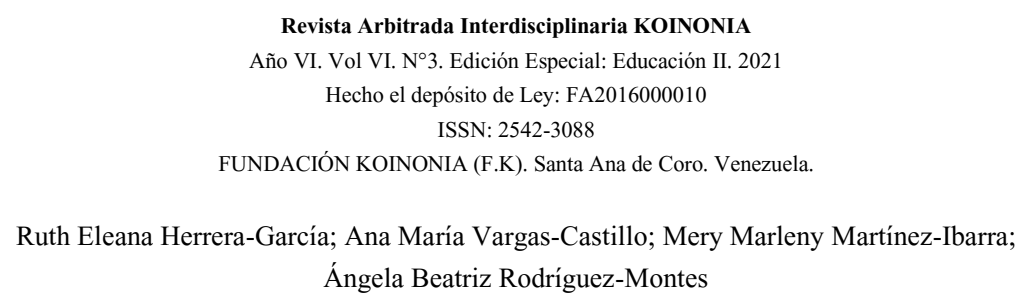

situaciones pedagógicas idóneas que conlleven a un exitoso proceso de enseñanza aprendizaje de estudiantes.

En tal sentido, y considerando los hallazgos encontrados en torno al análisis realizado sobre el desempeño del docente, se puede enunciar que, necesariamente el docente que aspira tener éxito en su proceso pedagógico, debe desplegar acciones concretas que lo muestren como un profesional de vanguardia involucrado con las TIC, para establecer nuevas formas didácticas de enseñar y motivar al aprendizaje.

Al mismo tiempo, en estos escenarios, debe saber gestionar espacios de buena escucha y diálogo, pues el proceso comunicacional y de interrelación, tanto con los estudiantes, como con los padres de familia, es fundamental para orientar y guiar las diversas situaciones sociales, psicológicas y emocionales, que puedan estar perturbando el proceso de acompañamiento pedagógico. En otras palabras, para coadyuvar a su desarrollo socioemocional; sobre todo, ahora, que la educación a distancia y basada en la virtualidad dejo de ser una elección para convertirse en la vía expedita para abordar contextos educativos y desarrollar escenarios de aprendizaje en situaciones adversas, como las que se viven a causa de la pandemia por Covid19.

\section{FINANCIAMIENTO}

No monetario.

\section{AGRADECIMIENTO}

A la Universidad Cesar Vallejo, Chimbote; por motivar el desarrollo y fomento de la investigación.

\section{REFERENCIAS CONSULTADAS}

Aguilar, M. (2014). Aprendizaje de conductas pro sociales desde la primera infancia como estrategia para el mejoramiento de la convivencia escolar y la prevención del "Bullying" (tesis de postgrado) [Learning pro-social behaviors from early childhood as a strategy for improving school coexistence and preventing "Bullying" (postgraduate thesis)]. Medellín, Colombia. Recuperado de https://n9.cl/ftzce 


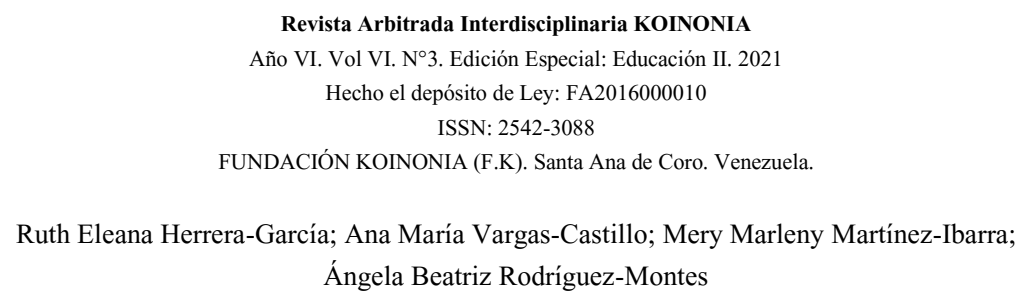

Cabañas, J. \& Ojeda, Y. (2003). Aulas virtuales como herramientas de apoyo en la educación de la Universidad Nacional Mayor de San Marcos (tesis de pregrado) [Virtual classrooms as support tools in education at the Universidad Nacional Mayor de San Marcos (undergraduate thesis)]. Universidad Nacional Mayor de San Marcos. Lima, Perú. Recuperado de https://n9.cl/pl4ax

Consuegra, N. (2010). Diccionario de Psicología [Psychology Dictionary]. Recuperado de https://n9.cl/3q7e1p

Covarrubias, C. \& Mendoza, M. (2013). La teoría de autoeficacia y el desempeño docente: el caso de Chile [The theory of self-efficacy and teacher performance: the case of Chile]. Estudios Hemisféricos y Polares. 4(2), 07-123.

Flores, F. (2008). Las competencias que los profesores de educación básica movilizan en su desempeño profesional docente (tesis de doctorado) [The competences that basic education teachers mobilize in their professional teaching performance (doctoral thesis)]. Universidad Complutense de Madrid, España. Recuperado de https://eprints.ucm.es/id/eprint/8171/1/T30412.pdf

Gálvez, E. \& Milla, R. (2018). Evaluación del desempeño docente: Preparación para el aprendizaje de los estudiantes en el Marco de Buen Desempeño Docente. Propósitos y Representaciones, 6(2), 407-452. Doi: http://dx.doi.org/10.20511/pyr2018.v6n2.236.

Garaigordobil, M. (2014). Conducta prosocial: el papel de la cultura, la familia, la escuela y la personalidad [Prosocial behavior: the role of culture, family, school and personality]. Revista Mexicana de Investigación en Psicología, 6(2), 146157.

Goleman, D. (1996). La inteligencia emocional: Por qué es más importante que el coeficiente intelectual [Emotional Intelligence: Why It Matters More Than IQ]. Recuperado de https://n9.cl/788g1

Martínez, G., Guevara, A. \& Valles, M. (2016). El desempeño docente y la calidad educativa [Teaching performance and educational quality]. Ra Ximhai, 12(6), 123-134.

Martínez, G., Esparza, A. \& Gómez, R. (2020). El desempeño docente desde la perspectiva de la práctica profesional [Teaching performance from the perspective of professional practice]. Revista RIDE, 11(21). Doi: https://doi.org/10.23913/ride.v11i21.703

Ministerio de Educación (2012). Marco de Buen Desempeño Docente [Good Teaching Performance Framework]. Recuperado de https://n9.cl/4g1e2 


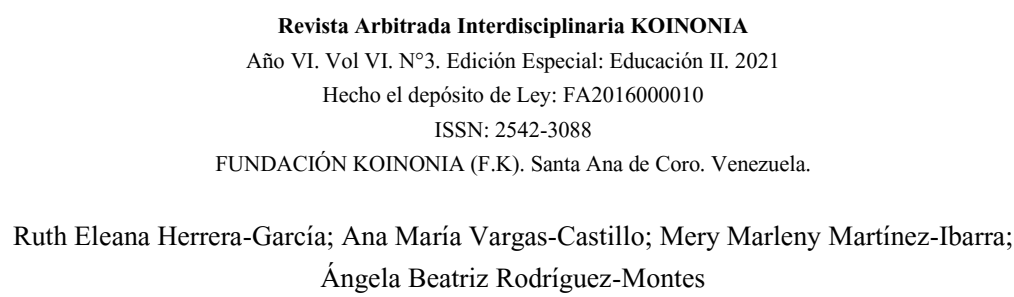

Palella, S. \& Martins, F. (2015). Metodología de la Investigación Cuantitativa [Quantitative Research Methodology]. Caracas. FEDUPEL.

Motta Ávila, J. H. (2017). La actitud de escucha, fundamento de la comunicación y la democracia en el aula [The attitude of listening, the foundation of communication and democracy in the classroom]. Cuadernos de Lingüística Hispánica, (30), 149-169. Doi: https://doi.org/10.19053/0121053X.n30.0.6192.

Rizo, M. (2020). Rol del docente y estudiante en la educación virtual [Role of the teacher and student in virtual education]. Revista Multi-Ensayos, 6(12), 28-37. Doi: https://doi.org/10.5377/multiensayos.v6i12.10117

Valdivieso, T. \& Gonzáles, M. (2016). Competencia Digital Docente: ¿Dónde estamos? Perfil del docente de educación primaria y secundaria. El caso de Ecuador [Teaching Digital Competence: Where are we? Profile of the teacher of primary and secondary education. The case of Ecuador]. Pixel-Bit. Revista de Medios y Educación, 49, 57-73. 